(e) A head CT scan would be a useful investigation

9 A 77-year-old woman with atrial fibrillation and a previous minor stroke presented to the clinic with breathlessness which was found to be caused by congestive cardiac failure. Her symptoms improved with diuretic therapy and her consultant then considered her future clinical management. National Institute for Health and Clinical Excellence guidelines indicate that:

(a) Her all-cause mortality risk would be reduced by anticoagulation therapy

(b) Her risk of an adverse outcome such as bleeding on anticoagulation therapy outweighs the potential benefit of stroke reduction

(c) She is at high risk of future thromboembolic stroke

(d) She should receive anticoagulation therapy

(e) The potential reduction of her risk of a future thromboembolic stroke outweighs her risk of an adverse outcome such as bleeding on anticoagulation therapy

10 An 82-year-old woman on longterm treatment for hypertension, myxoedema, dependent oedema secondary to venous insufficiency, and overactive bladder was admitted to hospital. She was pyrexial with severe below knee cellulitis. During the next two days she developed hyperactive delirium. Administration of her usual medication was difficult. Omission of the next few doses of the following medication may be associated with worsening of her agitation:
(a) Amlodipine
(b) Citalopram
(c) Furosemide
(d) Levothyroxine
(e) Oxybutynin

\section{Self-Assessment Questionnaire (SAQ) Acute Medicine CME}

\section{Clinical Medicine June 2007}

We have recently introduced the best of five format for the SAQs. This is in line with the changes introduced by the Federation of the Royal Colleges of Physicians of the UK and are now required in order to claim external CPD credits. This ideally should be accompanied by an expansion of the number of question but we are loath to increase the burden on our already hard-pressed authors. There have also been the technical teething problems (see below) for which we apologise. We plan to continue the new format and evaluate the outcome later this year. Any comments would be welcome and contribute towards finding an effective solution.

\section{Erratum}

Please note that the SAQ answers initially logged on 6 August, and published on page 407 of the August issue, were incorrect.

The correct answers are below. All submitted SAQs have now been remarked. Answers to the CME SAQs published in Clinical Medicine June 2007

$\begin{array}{llllllllll}\text { Q1 } & \text { Q2 } & \text { Q3 } & \text { Q4 } & \text { Q5 } & \text { Q6 } & \text { Q7 } & \text { Q8 } & \text { Q9 } & \text { Q10 } \\ \text { (a) } & \text { (b) } & \text { (e) } & \text { (b) } & \text { (d) } & \text { (e) } & \text { (e) } & \text { (a) } & \text { (a) } & \text { (d) }\end{array}$

Robert Allan Editor

\section{CME Cardiology SAQs}

\section{Answers to the CME SAQs published in Clinical Medicine August 2007}

$\begin{array}{llllllllll}\text { Q1 } & \text { Q2 } & \text { Q3 } & \text { Q4 } & \text { Q5 } & \text { Q6 } & \text { Q7 } & \text { Q8 } & \text { Q9 } & \text { Q10 } \\ \text { (e) } & \text { (c) } & \text { (d) } & \text { (d) } & \text { (b) } & \text { (d) } & \text { (b) } & \text { (a) } & \text { (a) } & \text { (e) }\end{array}$

\section{Errata}

\section{CME Cardiology SAQs. Clin Med 2007;7:405-7.}

Question 8. Please note that the haemoglobin level should be $13.2 \mathrm{~g} / \mathrm{dl}$ rather than $3.2 \mathrm{~g} / \mathrm{dl}$.

\section{Epstein M, Barmania N, Robin J, Harbord M. Reforming the} acute phase of the inpatient journey. Clin Med 2007;7:343-7.

Please note that the third author of this paper was incorrectly listed as J Robini in the table of contents and on the article title page. We apologise for any inconvenience caused. 